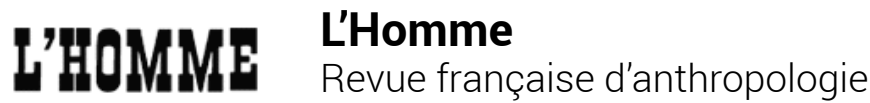

163 | juillet-sptembre 2002

De la légende au mythe. Parole, langue et pensée

\section{Christelle Violette-Bajard, Visage de la pauvreté. Don alimentaire et précarité urbaine}

Lyon, Chronique Sociale, 2000, 218 p.

\section{Noël Jouenne}

\section{(2) OpenEdition}

\section{Journals}

Édition électronique

URL : http://journals.openedition.org/lhomme/12711

DOI : 10.4000//homme.12711

ISSN : 1953-8103

Éditeur

Éditions de l'EHESS

Édition imprimée

Date de publication : 21 juin 2002

Pagination : 296-298

ISBN : 2-7132-1771-7

ISSN : 0439-4216

\section{Référence électronique}

Noël Jouenne, "Christelle Violette-Bajard, Visage de la pauvreté. Don alimentaire et précarité urbaine », L'Homme [En ligne], 163 | juillet-sptembre 2002, mis en ligne le 10 juillet 2007, consulté le 22 septembre 2020. URL : http://journals.openedition.org/lhomme/12711 ; DOI : https://doi.org/10.4000/ Ihomme.12711

Ce document a été généré automatiquement le 22 septembre 2020

(c) École des hautes études en sciences sociales 


\section{Christelle Violette-Bajard, Visage de la pauvreté. Don alimentaire et précarité urbaine}

Lyon, Chronique Sociale, 2000, 218 p.

\section{Noël Jouenne}

1 si faire de l'ethnologie c'est faire corps avec son terrain, alors Christelle Violette-Bajard répond à cette définition puisqu'elle a choisi de traiter de la pauvreté en étudiant les pratiques du don alimentaire. L'auteur a d'abord vécu une situation de précarité extrême avant d'en faire un objet de recherche. Elle s'en explique succinctement dès le début: "il ne s'agit pas de faire amende honorable, mais je tiens à souligner la sensation tenace de malaise de ce jeu de dupe sur les raisons de ma présence [...] Même si ma situation familiale, matérielle et financière n'était guère différente de la leur " (p. 25). L'implication est donc double, ce que le préfacier, Louis Moreau de Bellaing, ne manque pas de préciser.

2 Il s'agit d'un essai d'ethnologie suffisamment rare dans ce domaine pour être mentionné. Le contenu de l'ouvrage relève d'une forme adaptée au public visé par l'éditeur (travailleurs sociaux). D'où une didactique parfois pesante, comme la mise en surépaisseur de phrases ou de mots et les schémas de synthèse placés en fin de partie qui, à mon sens, troublent davantage la lecture qu'ils n'appuient l'argumentation. Ce genre d'artifice n'ajoute rien à la valeur du travail de l'ethnologue. En revanche, l'absence de bibliographie empêche toute tentative d'ouverture.

3 L'ouvrage se compose de deux parties. Dans la première, l'auteur offre une ethnographie fine du «rituel de la soupe populaire » à partir d'observations menées durant trois hivers au lieu de don alimentaire de l'église Saint-Eustache à Paris. L'auteur y décrit les ambiances, les attitudes, les postures, les attentes, les jeux de regard, les paroles durant tout le déroulement du repas. Le plus souvent, l'ethnologue s'efface derrière la troisième personne du singulier, créant ainsi une distance qui lui permet de construire son objet. Cette «neutralité» pose parfois question dans la mesure où le chercheur se trouve au cœur des interactions. 
C'est ainsi que Christelle Violette-Bajard va être initiée aux rites de cette culture de la rue et des soupes populaires. Cela commence par des mises «en garde contre le pas fatidique vers les oubliettes de la déchéance " (p. 24), à quoi succède la perception d'une solidarité. «Car la soupe est aussi un lieu de rencontre, de réseau de relations, de convivialité et de chaleur pour tout individu en rupture de ban, en désarroi affectif " (p. 53). Cette sorte de « rituel saisonnier » se caractérise par la recherche de l'anonymat pour chaque bénéficiaire, d'une solidarité pouvant rapidement faire volte-face, et par des rapports de pouvoir entre les bénévoles et les bénéficiaires. Malgré cela, l'auteur décrit les multiples visages qu'elle rencontre à travers ce qu'elle nomme une «pauvreté polymorphe ».

5 Sont ainsi distinguées plusieurs sous-catégories - «les anciens ruraux», «les honteux », «les rapaces »- qui sont raccrochées aux pratiques alimentaires en ce qu'elles ont de spécifique. Par exemple, «les "honteux" consomment intégralement la nourriture sur place» (p. 71); « les "jeunes nomades", en groupe, se distinguent par des habitudes alimentaires et une approche de la nourriture radicalement différentes » (p. 72). Il s'ensuit une analyse de la symbolique du don alimentaire que l'auteur met en relation avec les mécanismes du rite de passage. «La soupe s'inscrit donc comme le support symbolique d'un passage vers un statut supérieur similaire à celui du donateur » (p. 77). Réinvestissant le lieu de don aux côtés des bénévoles, l'ethnologue découvre leur regard, leur rapport à la souillure et à une certaine violence symbolique. Les interactions entre bénévoles et «bénéficiaires » suscitent de multiples échanges silencieux qui sont, pour l'auteur, autant de non-dits qui permettent l'acceptation d'une "violence silencieuse » contribuant à un rééquilibrage des forces au niveau collectif : «À l'humiliation de n'être qu'une main tendue, les exutoires de cette violence silencieuse sont donc aussi silencieux» (p. 99). Christelle Violette-Bajard consacre quelques sous-chapitres à l'étude de la transmission des valeurs par la parole, ou par l'absence de parole, qui vont contribuer à créer un sentiment d'appartenance à un groupe plutôt qu'à une catégorie. C'est ainsi que «les novices sont immédiatement repérés car ils déteignent dans le paysage conventionnel » (p. 107). L'apprentissage des valeurs du groupe participe d'un ensemble de savoir-faire acquis sur le tas.

6 La seconde partie est consacrée à une analyse de la pauvreté du point de vue historique et sociologique à partir des éléments recueillis. L'auteur se réfère aux grands classiques en la matière (Michel Foucault, Bronislaw Geremek, Michel Mollat, Pierre Sassier, Alexis de Tocque-ville), ce qui la conduit à amorcer une réflexion à partir des travaux, devenus des classiques, de René Girard et de Colette Pétonnet sur le sacrifice. On regrettera, de ce point de vue, l'absence de l'apport ethnologique de ces dix dernières années (Carole Amistami, Patrick Gaboriau, Daniel Terrolle) ${ }^{1}$ qui aurait permis une mise en perspective et une comparaison actuelle, notamment en ce qui concerne le don alimentaire.

7 Au terme de cet essai, Christelle Violette-Bajard est amenée à considérer la logique du don alimentaire comme faisant partie d'un processus sacrificiel : «La société semble donc produire "un mal" qui lui est nécessaire, une catégorie sacrifiée et sacrifiable à merci » (p. 212). Comme je l'ai moi-même observé2 « «la période à laquelle se déroule ce rituel n'est pas sans importance» (p. 214), ce qui conforte l'hypothèse du rythme saisonnier comme faisant partie du rituel. Dans sa conclusion, l'auteur esquisse une ethnologie du don alimentaire en montrant comment l'instauration hivernale du « rituel des soupes populaires » (p. 216) relève de cette logique sacrificielle. 
Enfin, on notera le côté « engagé » du chercheur qui, par ses prises de position, relance le débat pour une recherche impliquée : «La misère de notre société réside aussi dans celle de ces politiques de tout poil qui n'ont ni le courage ni la volonté du respect des droits de l'homme et qui tirent leur révérence à la pensée unique » (p. 218). Du fait de sa double implication, l'ethnologue est ici en prise directe avec la pauvreté, dont cet ouvrage est un témoignage éclairé.

\section{NOTES}

1. Cf. Carole Amistami, «À la soupe... » Ethnologie du don alimentaire envers les populations défavorisées. L'alimentation des Sans Domicile Fixe, Mémoire de maîtrise s. dir. Daniel Terrolle, Université Paris VIII, 1992, et «L'alimentation des SDF : l'exemple parisien », in Daniel Terrolle, s. dir., "Errances urbaines ». Recherche en ethnologie urbaine, Rapport Plan Urbain, 1993.

2. «Ainsi, les périodes hivernales semblent être les moments de l'année choisis pour [le] sacrifice [des SDF] » (Noël Jouenne, Approche de la problématique sine domo en Haute-Loire et au Puy-en-Velay du milieu du XIXe siècle à nos jours : contribution à l'étude du vagabondage, Thèse de doctorat, Université René Descartes, Paris, 1997 : 765).

\section{AUTEUR}

\section{NOËL JOUENNE}

CNRS, Laboratoire d'anthropologie urbaine, Ivry-sur-Seine. 